\title{
Investigação de hemoglobinopatias em sangue de cordão umbilical de recém-nascidos do Hospital de Base de São José do Rio Preto
}

\author{
Ricardo P. Ducatti \\ Antonio E.A. Teixeira ${ }^{1}$ \\ Heloísa A. Galão ${ }^{2}$ \\ Claudia R. Bonini-Domingos ${ }^{3}$ \\ Agnes C. Fett-Conte
}

\begin{abstract}
As hemoglobinopatias são as doenças genéticas mais freqüentes na população bumana. Cerca de 12 a 15\% da população é portadora de uma ou mais formas de hemoglobinas anormais, resultando em um grande problema de saúde pública. O diagnóstico neonatal possibilita o tratamento e o aconselhamento genético precoce, incluindo a conscientização dos portadores sobre o risco do nascimento de homozigotos. O objetivo deste trabalho é apresentar os resultados da investigação de hemoglobinopatias em recémnascidos do Hospital de Base de São José do Rio Preto. O estudo foi realizado em 913 amostras de sangue de cordão umbilical, que foram submetidas a testes eletroforéticos, bioquímicos e citológicos, especificos para análise de hemoglobinas. Foram identificadas 100 (10,95\%) amostras com hemoglobinas anormais, das quais 40 (4,38\%) com Hb Bart's, sugerindo alfa talassemia, 34 (3,72\%) com $\mathrm{Hb}$ S, 23 (2,52\%) com beta talassemia, duas (0,22\%) com Hb Ce uma $(0,11 \%)$ amostra apresentou $\mathrm{Hb}$ rápida. A frequência elevada de alterações encontradas evidencia a necessidade da triagem neonatal de hemoglobinopatias que pode resultar em amplos beneficios para os portadores destas patologias e seus familiares. Rev.bras.hematol.hemoter., 2001, 23(1): 23-29
\end{abstract}

Palavras-chave: Hemoglobinopatias, diagnóstico neonatal

\section{Introdução}

Entre as doenças genéticas, as hemoglobinopatias são as mais freqüentes na população, especialmente as talassemias e a anemia falciforme, consideradas um grande problema de saúde pública. De 12 a 15\% da população humana é portadora de uma ou mais formas de anemias hereditárias, com conseqüências que variam de imperceptíveis até letais $(1,2)$.
A anemia falciforme é a hemoglobinopatia com maior incidência na população brasileira. Sua etiologia é uma mutação que ocorre no gene da globina beta, que produz uma hemoglobina anormal, denominada hemoglobina S (HbS). Clinicamente, o portador pode apresentar-se como assintomático (forma heterozigota, com a presença de um único gene mutado) ou sintomático (forma homozigota, com a presença dos dois genes mutados). A cristalização da HbS tem como conseqüências

\footnotetext{
1. Serviço de Genética. Departamento de Biologia Molecular. Faculdade de Medicina de São José do Rio Preto (FAMERP)

2. Departamento de Ginecologia e Obstetrícia. FAMERP

3. Centro de Referência de Hemoglobinas. UNESP
}

Correspondência para: Agnes C. Fett-Conte

Serviço de Genética - FAMERP. Av. Brigadeiro Faria Lima, 5416. CEP: 15090-000. São José do Rio Preto. SP. Brasil E-mail: genetica@famerp.br 
principais a anemia hemolítica crônica e a oclusão de pequenos vasos sangüíneos, que resultam em lesão tecidual isquêmica com crises de dor, infartamento e necrose em diversos órgãos. A doença é predominante entre negros e pardos. Estimativas com base na prevalência admitem, no Brasil, a existência de mais de dois milhões de portadores do gene da HbS, aproximadamente oito mil afetados na forma homozigota e o nascimento de 2500 casos novos por ano (3, 4, 5, 6, 7, 8, 9, 10).

As síndromes talassêmicas, por sua vez, compreendem um grupo heterogêneo de distúrbios genéticos, caracterizados pela ausência ou redução na síntese de cadeia globínica. A talassemia beta caracteriza-se pela síntese deficiente de cadeia beta e a talassemia alfa, pela diminuição na síntese de globina alfa. Nas formas sintomáticas, as talassemias se apresentam como anemia hemolítica crônica e são, muitas vezes, fatais na infância $(11,12,13,14,15,16,17)$.

Considerando a freqüência das hemoglobinopatias no Brasil e a gravidade de certas formas dessa patologia, o diagnóstico precoce é fundamental uma vez que, por exemplo, cerca de $20 \%$ das crianças portadoras de anemia falciforme não chegam aos cinco anos de idade e o restante apresenta uma redução acentuada do rendimento escolar. Se diagnosticada precocemente, a criança poderá receber tratamento adequado nos primeiros anos de vida, reduzindo as complicações que decorrem da doença $(3,18,19)$.

Dados da literatura mostram que o diagnóstico precoce, sobretudo ao nascimento, e o tratamento adequado, melhoram drasticamente a taxa de sobrevida e a qualidade de vida dos doentes. Na anemia falciforme o simples esquema de vacinação contra pneumococo e Haemophilus influenza, acompanhado de penicilinoterapia profilática, diminui o número de mortes no período crítico, situado entre os seis meses e os três anos de idade $(20,21,22,23,24,25,26)$.

Considerando as formas graves das hemoglobinopatias, a freqüência elevada de heterozigotos e a possibilidade de tratamento precoce, a triagem neonatal destas patologias é importante para a comunidade. Inclusive, a criação de programas desta natureza tem sido estimulada por várias entidades internacionais, como a Organização Mundial de Saúde, a Academia de Ciências do Terceiro Mundo e a Organização Panamericana da Saúde (21, 27, 28, 29). Entretanto, até o presente, não há descrição de trabalhos que envolvam a triagem neonatal destas patologias na região de São José do Rio Preto, SP.

Neste contexto, o presente estudo teve como objetivo investigar a presença de hemoglobinopatias em amostras de sangue de cordão umbilical de recém-nascidos do Hospital de Base de São José do Rio Preto.

\section{Casuística e Métodos}

Este estudo, aprovado pelo Comitê de Ética em Pesquisa da Faculdade de Medicina de S.J. do Rio Preto (FAMERP), foi realizado em amostras de sangue de cordão umbilical de recém-nascidos do Hospital de Base de São José do Rio Preto, SP, no período de maio de 1997 a setembro de 1998.

A coleta do sangue do cordão umbilical foi efetuada pelos obstetras responsáveis pelos partos, de acordo com protocolo de rotina para tipagem sanguínea (ABO e Rh), e as amostras foram encaminhadas diretamente para $O$ Serviço de Genética. Amostras de $5 \mathrm{ml}$ de sangue foram coletadas em tubo "vacutainer" com EDTA e submetidas aos seguintes testes seletivos: resistência globular osmótica em $\mathrm{NaCl}$ a 0,36\% (30), análise a fresco da morfologia eritrocitária e eletroforese em pH alcalino (31). Os casos suspeitos de apresentar alteração de hemoglobinas foram submetidos a testes para confirmação, que incluíram: dosagem de hemoglobina $\mathrm{A}_{2}$ (31), pesquisa de hemoglobina $\mathrm{H}$ intraeritocitária (32), dosagem de hemoglobina fetal (33) e eletroforese em ágar fosfato pH 6,2 $(34,35)$.

Para a confirmação diagnóstica, os neonatos diagnosticados na triagem como portadores de hemoglobinopatias, foram avaliados após o sexto mês de vida (27). Com a confirmação da hemoglobinopatia, os mesmos foram encaminhados aos serviços de HematoPediatria e de Genética para avaliação clínica, tratamento e aconselhamento genético. 


\section{Resultados}

Foram analisadas 913 amostras de sangue de cordão umbilical no período de maio de 1997 a setembro de 1998. Os resultados obtidos mostraram a presença de hemoglobinas anormais em 100 amostras $(10,95 \%)$. Destas, 40 (4,38\%) apresentaram Hb Bart's, que sugere alfa talassemia, $34(3,72 \%)$ apresentaram traços de $\mathrm{HbS}, 23$ (2,52\%) foram sugestivas de beta talassemia, duas $(0,22 \%)$ mostraram a presença de HbC e uma amostra $(0,11 \%)$ apresentou $\mathrm{Hb}$ rápida. Esses resultados são apresentados na Tabela 1.

Tabela 1. Freqüências de bemoglobinas anormais encontradas em amostras de sangue de recém-nascidos

\begin{tabular}{c|c|c|}
\hline \multirow{2}{*}{ Tipo de hemoglobina } & \multicolumn{2}{|c}{ Freqüência } \\
\cline { 2 - 3 } & $\mathbf{n}^{\mathbf{o}}$ & $\mathbf{\%}$ \\
\hline Hb normal (AF) & 813 & 89,05 \\
Hb Bart's (AF + Bart's) & 40 & 4,38 \\
\hline Hb S (AFS) & 34 & 3,72 \\
Beta talassemia (F)* & 23 & 2,52 \\
\hline Hb C (AFC) & 02 & 0,22 \\
Hb rápida (AF + ?) & 01 & 0,11 \\
\hline TOTAL & 913 & 100 \\
\hline
\end{tabular}

* Ausência de HbA e prevalência de HbF

\section{Discussão}

Para o diagnóstico neonatal utiliza-se, geralmente, o sangue de cordão umbilical, e nesta fase de vida, os componentes hemoglobínicos que predominam são a $\mathrm{HbF}$ (90 a 100\%), a HbA (0 a 10\%) e a $\mathrm{Hb} \mathrm{A}_{2}(0$ a 1\%). Após o nascimento e até aproximadamente seis meses de vida haverá a inversão na produção das cadeias hemoglobínicas, podendo ser observados os valores definitivos do indivíduo adulto: $\mathrm{HbA}$ (96 a 98\%), $\mathrm{HbA}_{2}$ (2,5 a 3,7\%) e $\mathrm{HbF}$ (0 a 1\%). Para o diagnóstico neonatal, principalmente nas alterações de cadeia beta, como é o caso das falcemias, só são encontrados os traçados eletroforéticos característicos após o sexto mês de vida. Na fase neonatal apenas traços das hemoglobinas anormais de cadeia beta podem ser visualizados (11).

Para programas preventivos de anemia falciforme em neonatos, são utilizadas eletroforeses alcalina e ácida. Os testes de solubilidade para a triagem de HbS são desaconselhados, uma vez que, estes não permitem distinguir indivíduos AS, SS ou SC. Além disso, em neonatos, estes testes costumam ser negativos devido à pequena concentração da HbS. Entretanto, podem ser utilizados como testes de confirmação após a eletroforese alcalina $(11,13)$.

As hemoglobinopatias têm sido muito estudadas no Brasil quanto à sua distribuição e incidência em diferentes regiões e grupos

Tabela 2 . Freqüências de hemoglobinopatias em recém-nascidos obtidas em trabalhos realizados em Londres (Inglaterra)(36) e Togo (África)(40)

\begin{tabular}{l|c|c|c|c}
\hline \multirow{2}{*}{} & \multicolumn{2}{|c|}{ Togo } & \multicolumn{2}{c}{ Londres } \\
\cline { 2 - 5 } & $\mathbf{n}^{\mathbf{0}}$ & $\mathbf{\%}$ & $\mathbf{n}^{\mathbf{0}}$ & $\mathbf{\%}$ \\
\hline Hb normal & 240 & 61,8 & 931 & 93,1 \\
$\mathrm{Hb} \mathrm{S}$ & 81 & 21,0 & 26 & 2,6 \\
Beta talassemia & 4 & 1,0 & \multicolumn{2}{|c}{ Não analisada } \\
Hb Bart's & 22 & 5,7 & 21 & 2,1 \\
Hb C & 40 & 10,5 & 12 & 1,2 \\
Outros tipos de Hb & & & 10 & 1,0 \\
Total & 387 & 100 & 1.000 & 100 \\
\hline
\end{tabular}


raciais, mas não em relação ao diagnóstico neonatal. Inclusive, a quantidade de trabalhos que abordam este tema, apesar de sua importância, é muito pequena na literatura nacional. O presente trabalho, portanto, é um dos poucos realizados no Brasil e o primeiro na região de São José do Rio Preto.

Há, entretanto, um volume substancial de publicações relacionadas ao diagnóstico neonatal das hemoglobinopatias em outros países, principalmente europeus, onde a triagem de hemoglobinopatias já é rotina no sistema de saúde (27). Estes trabalhos revelam incidências maiores ou menores de hemoglobinopatias em função da variabilidade étnica das populações estudadas. A Tabela 2, por exemplo, mostra a variação das freqüências de hemoglobinopatias entre uma população européia (36) e uma africana (40). Apesar desta variação, todos os trabalhos enfatizam a viabilidade, importância e os benefícios de programas de triagem neonatal em especial para as formas homozigotas, onde a intervenção clínica precoce, favorece a melhoria na qualidade de vida do doente $(36,37,38,39,40,41,42)$.

$\mathrm{Na}$ literatura relativa aos dados brasileiros, foram descritos dois trabalhos de diagnóstico neonatal de hemoglobinopatias em recém-nascidos, um no Serviço de Genética e Neonatologia da Universidade Federal da Paraíba (27) e outro no Centro da Fundação de Hemoderivados e Hemoterapia de Minas Gerais (43).

O trabalho realizado na Paraíba analisou 1006 amostras de sangue de recém-nascidos da zona urbana de João Pessoa e obteve os seguintes resultados: hemoglobina normal em 99,85\% dos casos, $\mathrm{Hb} \mathrm{S}$ em $0,1 \%$ e $\mathrm{Hb} \mathrm{C}$ em $0,05 \%$. Neste trabalho não foi investigada a presença de talassemias e os autores enfatizam as dificuldades de identificação das frações de hemoglobinas anormais pela metodologia utilizada (eletroforese em agar-amido), que pode ser utilizada apenas em uma triagem inicial (27).

A análise realizada em Minas Gerais, teve como objetivo principal a pesquisa de anemia falciforme em recém-nascidos. Os autores estudaram amostra de sangue de cordão umbilical de 16.935 recém-nascidos de 688 cidades mineiras. O resultado encontrado foi de 3,41\% para traços de anemia falciforme (HbS) e 1,32\% para HbC (43).

As diferenças entre os resultados aqui obtidos e os dos dois trabalhos brasileiros, podem ser explicados pela variabilidade étnica das populações estudadas e metodologia aplicada, principalmente no que se refere às talassemias em suas diferentes formas, que não foram avaliados pelos outros autores. Pela constituição racional da população brasileira, a maioria dos artigos sobre hemoglobinopatias no Brasil enfocam, principalmente, a hemoglobina S. Sua maior freqüência é observada nas regiões norte e nordeste, que sofreram grande influência da raça negra na constituição étnica de sua população. Além disso, as implicações clínicas justificam esta preocupação, devido, especialmente, aos gastos envolvidos no tratamento de portadores sintomáticos e sobrevida dos doentes (44).

Com relação a taxa de HbS encontrada, os dados obtidos em São José do Rio Preto foram compatíveis com os do trabalho desenvolvido em Minas Gerais (3,72\% e o 3,47\%, respectivamente), o que pode ser explicado pela semelhança na composição racial dos dois estados (10).

A hemoglobina variante do tipo C é um tipo de hemoglobina que desencadeia a formação de cristais no interior da hemácia. Da mesma forma que a $\mathrm{HbS}$, a HbC é característica de povos de origem africana, sendo encontrada com freqüências entre 5 e $25 \%$ em muitas regiões da África. A freqüência de HbC e de HbS $(0,11 \%$ e $3,72 \%$ respectivamente), em nossa população demonstra a integração do componente negróide na região $(10,42,43)$.

As talassemias, nas formas alfa e beta heterozigotas, foram as alterações mais freqüentes no presente trabalho (63\%). Esta incidência pode ser explicada pela colonização do estado de São Paulo. A região de São José do Rio Preto recebeu, durante o seu processo de formação populacional, significativo contingente de espanhóis, seguido por italianos, portugueses e, posteriormente, outros grupos europeus e asiáticos, com suas contribuições genéticas características, incluindo mutações nos genes codificadores de hemoglobinas (10, 44, 45).

Este trabalho detectou 100 recém-nascidos com anemias hereditárias, que podem ser 
potencialmente patológicas e cujos graus de comprometimento clínico dependem da constituição do genótipo de cada um. Com o diagnóstico no início de suas vidas, essas crianças têm a possibilidade de receber tratamento precoce e a família, orientação e aconselhamento genético acerca dessas alterações hereditárias $(28,42,43)$.

\section{Conclusão}

A incidência de hemoglobinopatias entre os 913 recém-nascidos estudados foi alta (10,9\%) e mostrou a necessidade da triagem neonatal na população. Este tipo de triagem tem custo baixo, portanto, pode ser incluída em programas de saúde e incorporada às rotinas hospitalares, possibilitando a prevenção, a orientação médica e genética para os afetados e seus familiares, aumentando a sobrevida e melhorando a qualidade de vida dos pacientes.

\section{Hemoglobinopathies in new-born cord blood of the Hospital de Base of Sao Jose do Rio Preto, SP - Brazil}

Ricardo P. Ducatti, Antonio E.A. Teixeira, Heloísa A. Galão, Claudia R. Bonini-Domingos, Agnes C. Fett-Conte

\footnotetext{
Abstract

Hemoglobinopathies are the most frequent genetic diseases in the human population. About 12 to 15\% of the population have one or more forms of abnormal hemoglobins, which creates a buge public health problem. The neonatal diagnosis permits early treatment and genetic counselling also makes carriers aware of the risk of homozygote children. The purpose of this study is to present the results of the investigation of hemoglobinopathies in newborn babies in the Hospital de Base, São José do Rio Preto. The study was performed on 913 blood samples taken from umbilical cords, which were submitted to specific electrophoresis, biochemical and cytologic tests for hemoglobin analysis. The results showed one bundred samples (10.95\%) bad abnormal hemoglobins of which 40 (4.38\%) were Hb Bart's suggesting alpha thalassaemia, 34 (3.72\%) were $\mathrm{Hb} S, 23$ (2.52\%) were Beta thalassaemia, two
}

(0.22\%) were Hb C and one (0.11\%) was fast bemoglobin. The bigh rate of abnormal bemoglobins shows the necessity of neonatal screening which can result in great benefits for carriers of these diseases and their relatives.

Rev.bras.hematol.hemoter., 2001, 23 (1): 23-29

Key words: Hemoglobinopathies, neonatal diagnosis

\section{Referências Bibliográficas}

1- Domingos C.R.B. Tese apresentada para obtenção de grau de Doutor em Ciências Biológicas, no curso de Pós- Graduação em Ciências Biológicas, Área de Concentração em Genética. Instituto de Biociências, Letras e Ciências Exatas, UNESP, Campus de São José do Rio Preto, SP, 1993.

2- Paiva e Silva R.B., Ramalho A.S., Cassorla R.M.S. A anemia falciforme como problema de saúde pública no Brasil. Rev. Saúde Públic; 1993. 27: 54-58.

3 - Ministério da Saúde, Coordenação de Sangue e Hemoderivados. Programa de anemia falciforme, Portaria MS No. 951 de 10/05/1996.

4 - Brandalise S.R., Tricta JR D.F. Criação de um centro abrangente de atenção ao doente falcêmico: um desafio num país subdesenvolvido. Centro de pesquisa oncobematológica da infância (CIPOI). Universidade de Campinas, SP. (Divulgação interna), 1996.

5 - Robbins S.L, Cotran R.S., Kuman V. Doença dos eritrócitos e distúrbios hemorrágicos. In:

Patologia Estrutural e Funcional. 4.ed. Rio de Janeiro: Guanabara Koogan, 1991. p. $540-576$.

6 - Cerqueira R., Gallo H.H.A. Manifestações clinicas e laboratoriais da doença falciforme nos pacientes do IEHE - HEMORIO. Bol. Soc. Brasil. Hematol. Hemoter.; 8, supl., 1996.

7 - Friedrish J.R. Acidente vascular cerebral isquêmico em jovem branco portador de traço falciforme, Bol. Soc. Brasil. Hematol. Hemoter.; 8, supl., 1996.

8 - Streetly A., Maxwell K., Campbell B. Cordinated neonatal screeniing programme for haemoglobin disorders is needed. BMJ; 21, 1998.

9- Lorey F.W., Arnopp J., Cunningham G.C. 
Distribution of hemoglobinopathiey variants by ethnicity in a multiethnic state. Genetic Epidemiology; 1996. 13(5): 501-502.

10 - Naoum P.C. et al. Hemoglobinas anormais no Brasil. Prevalência e distribuição geográfica. Rev.Bras.Pathol.Clin.; 1987. 23: 68-79.

11 - Naoum P.C. Talassemias. In: Diagnóstico das Hemoglobinopatias. Ed. São Paulo: Sarvier, 1987. p.99-117.

12 - Frank W., Hall L.C.D.R., et al. Screening for alfa thalassemia in neonates. Amer.J. Clin. Pathol., 1987. 87(3): 389-391.

13 - Dumars K.W., Boehm C., Eckman J.R. Pratical guide to the diagnosis of thalassemia. Council of Regional Networks for Genetics Services (CORN). Am.J.Med.Genet., 1996. 62(1): 29-37.

14 - Kazazian H.H.J. The thalassemia syndromes: molecular basis and prenatal diagnosis in 1990. Seminars in Hematology, 1990. 27 (3): 209-228.

15 - Golgdfarb A., Grisaru D., Gimmon Z., Okon E., Lebensart P. High incidence of chololithiasis in older patients with homozygous beta thalassemia. Acta Hematol; 1990. 83: 120-122.

16 - Cao A., Rosatelli B.S., Pirastu M. Thalassemias in Sardina: molecular pathology, phenotypegenotype correlation, and prevention. Am. J. Pediatric Hematology and Oncology; 13 (2): p. 179-188.

17- Rund D., Filon D., Strauss N., Rachmilewitz E.A. Mean corpuscularvolume of heterozygotes for beta thalassemia correlates with the severity of mutations. Blood, 1992. 79 (1): 238-243.

18- Lin T., Eng H.L., Kuo P.L. Neonatal screening for alpha-thalassemia in Southern Taiwan. Journal Formosan Med. Assoc.; 1992.91 (12).

19- Gessner B.D., Teutsch S.M.; Shaffer P.A. A cost-effectiveness evaluation of newborn bemoglobinopathy screening from the perspective of state health care systems. Early Human Developtment; 1996. 45: 257-275.

20. Smith A.J., Kidney R.T. Sickle cell disease: Screening and management in newborn and infants. American Family Physician, Julho, 1993. 48(1): 95-102.

21. Zhang Y.H., Mccabe L.L., Wilborn M. Application of molecular genetics in public health: improved follow-up in a neonatal bemoglobinopathy screening program. Biochem. Med. Metab. Biol.; 1994. 52 (1): 27-35.

22. Tsevat J.; Wong J.B.; Pauker P.G.; Steinberg M.H. Neonatal screening for sickle cell disease: a cost-effectiveness analysis. The Journal of Pediatrics; 1991. 118 (4).

23. Committee On Genetics. Health supervision for children with sickle cell diseases and their families. American Academy of Pediatric, 1996. 98 (3).

24. Silveira E.P., Rocha A.F. Aconselhamento genético: estratégia de prevenção para as hemoglobinopatias. Bol. Soc. Brasil. Hematol. Hemoter., v.8, supl. Outubro, 1996.

25. Gribbons D., Zahr N.K., Opas S.R. Nursing menagement of children with sickle cell disease: an update. J. Pediatr. Nurs; 1995. 10 (4), 232-242.

26. Ferreira C., Piccoli A.C.M., Camanho, D. Avaliação das medidas profiláticas na doença falciforme. Bol. Soc. Brasil. Hematol. Hemoter., Outubro, 1996. v.8, supl.

27. Pantaleão S.M., Medeiros J.G., Nunesmaia H.G. Triagem de hemoglobinopatias estruturais em recém-nascidos de João Pessoa - PB. Rev.Bras.Pat.Clin.; 1993. 29 (1): 8-13.

28. Compri M.B., Polimeno N.C., Stella M.B., Ramalho A.S. Programa comunitário de bemoglobinopatias hereditárias em população estudantil brasileira. Rev.Saúde Pública; 1996. 30 (2): 187-95.

29. Jornal Hemominas. Teste do pezinho, programa garante a realização do exame no Estado. Jornal Hemominas. Órgão informativo da Fundação Centro de Hematologia e Hemoterapia de Minas Gerais, Fev. 1998. Ano VIII, 36, 3-6.

30. Silvestroni E. \& Bianco I. Screening for microcytemia in Italy: analysis of data collected in the past 30 years. Am. J. Hum. Genetics; 1975. 27: 198.

31. Marengo-Rowe A.J. Rapid electrophoresis and quantitation of haemoglobin on cellulose acetate. J.Clin.Path.; , 1965. 18: 90-792.

32. Papayannopoulous R. \& Stamatayannopoulous G. Stains for inclusion bodies. "In: Standartization of laboratory reagents and methods for detection of haemoglobinopathies". Hew Publications, (CDC) Atlanta, Georgia. 1974. 
33. Betke, K.; Marti, N. R. \& Schlicht, I. Estimation of small percentages of phoetal hemoglobin. Nature; 1959. 184:1877.

34. Vella F. Acid agargel electrophoresis of human hemoglobin. Am.J.Clin.Path.; 1968. 49: 440.

35. Naoum P.C. Eletroforese, técnica e diagnóstico. Ed. Santos, SP, 1990.

36. Henthorn J., Anionwu E., Brozovic M. screening cor blood for sickle haemoglobinopathies in Brent. British Medical Journal; 1984. 289: 479-80.

37. Githens J.H., Peter L., Mccurdy R.S. Newborn screening for hemoglobinopathies in Colorado. AJDC; 1990. 144.

38. West R., Ashcraft P., Becton D. Newborn screening for hemoglobinopathies in Arkansas: First Two Years' Experience. Journal of the Arkansas Medical Society; 1992. 88 (8).

39. Shafer F.E., Lorey F., George C.C. Newborn screening for Sickle cell disease: 4 years of experience from California's Newborn screening program. Journal of Pediatric Hematology Oncology; 1996. 18, (1): 36-41.

40. North M.L., Piffaut M.C. Detection of haemoglobinopathies at birth in Togo. Rev
Fr Hematology; 4: 237-241, 1988.

41. Cabot D.A., Casado T.D., Barbean P.J., Roqueta S.M. Neonatal screening for sickle cell disease in the Consorci Sanitari de Mataro.Rationale and first results. Esp Pediatric; 1998. 4(2):157-60.

42. Grossman L.K. Neonatal screening and genetic counseling for sickle cell trait. AJDC; 1985. 139.

43. Jornal Hemominas. Programa Triagem Neonatal apresenta primeiros resultados. Jornal Hemominas, Orgão informativo da Fundação Centro de Hematologia e Hemoterapia de Minas Gerais, 1998. ano VIII, n.37, p.4-5, março-abril.

44. Naoum P.C., Angulo I.L., Brandão A.C. Detecção e conscientização de portadores de hemoglobinopatias nas regiões de São José do Rio Preto e Presidente Prudente, SP (Brasil).

Rev.Saúde Pública; 1985. 19: 364-73.

45. Naoum P.C. Anemias imigrantes, origem das anemias hereditárias no Brasil. Ciência Hoje; 1984. set/out. 3 (14).

Recebido: 08/07/00

Aceito: 10/12/00 